\title{
Perfiles de ocio deportivo en jóvenes españoles (15-20 años): un análisis de género Sports leisure profiles in Spanish youth (15-20 years): a gender analysis
}

\author{
Raúl Fraguela-Vale, Lara Varela-Garrote, Laura Varela-Crespo
}

Universidad de La Coruña (España)

Resumen. El objetivo de este artículo es analizar las características de la práctica físico-deportiva durante el ocio de la juventud que cursa Educación Secundaria Postobligatoria no universitaria (ESPO) en España (de edades entre 15 y 20 años). Concretamente, se estudia la práctica de ocio deportivo en función del género y se analizan las características de la misma (frecuencia, organización, compañía, etc.). Para ello se realizó un diseño de investigación cuantitativo en el que se aplicó un cuestionario elaborado ad hoc a una muestra representativa de 2694 estudiantes españoles de ESPO, que señalaron el ocio deportivo como su opción preferida de ocio (n= 901). En general, la modalidad de ocio preferida por la juventud española es la deportiva. Las chicas se decantan por actividades físicas individuales y artístico-expresivas, mientras que los chicos por la práctica de deportes colectivos; siendo menor el interés de las chicas por participar en competiciones deportivas. Además, la frecuencia de práctica es inferior en las mujeres durante la semana, pero no durante los fines de semana. Ellas renuncian en mayor medida que ellos a practicar actividades de ocio que les gustan. Respecto a la dimensión social de la práctica, ambos colectivos realizan deporte mayoritariamente con gente de su edad, pero ellas puntúan más bajo en esta opción de respuesta. El género es un factor determinante en la práctica de ocio deportivo juvenil, constatándose desigualdades en la frecuencia y preferencias de práctica y en la renuncia a actividades físico-deportivas que son de su agrado.

Palabras clave: ocio, deporte, género, actividad de tiempo libre, juventud.

Abstract. We study the profiles of sports participation of youths enrolled in Post-Compulsory Secondary Education (PCSE) in Spain (15-20 years). Specifically, it is analyzed if there are any differences according to gender in sports leisure practice and what are the sport participation features of each group. In order to do so, a quantitative research design was carried out and an ad hoc questionnaire was applied to a significative sample of 2694 ESPO Spanish students. Nine hundred and one of them were specifically studied because they selected sports practice as their main leisure preference. Overall, it is observed that the kind of leisure activity chosen by the Spanish youth is sport activity. We found that girls prefer individual and artistic-expressive physical activities, while boys opt for practicing team sports. We found a lower interest of girls in participating in sports competitions. Also, the frequency of practice is lower in women than men during the working days, but not during the weekend. Girls give up practicing leisure activities that they like much more than boys do. Regarding the social dimension of the practice, both groups practice mostly with people of their age, but girls score lower in this response option. Gender affects sports leisure of Spanish youth, differences being found in the frequency and preferences of practice, and in waivering leisure activities that they like.

Keyword: leisure, sport, gender, leisure time activities, youth.

\section{Introducción}

La tarea anunciada décadas atrás por Dumazedier (1964) de hacer del ocio un tiempo educativo continúa despertando el interés de los Estudios de Ocio que, desde una perspectiva interdisciplinar, han profundizado en temáticas específicas como la planificación y organización de los espacios (Ortega, Lazcano \& Rocha, 2015), los itinerarios de los hobbies y aficiones (Monteagudo \& Cuenca, 2012), los beneficios individuales y colectivos (Monteagudo, Ahedo \& Ponce de León, 2017), etc., evidenciando la relevancia del ocio como factor de desarrollo humano.

Existen tantos estilos de ocio como personas, puesto que se trata de una experiencia de carácter subjetivo y personal que tiene diversos sentidos y significados (Ureta, 2015), pero la experiencia de ocio alcanza también una dimensión social que resulta fundamental para la etapa juvenil, en la medida en que se comparten intereses y se desarrollan proyectos comunes especialmente con el grupo de iguales. Diversas actividades como practicar un deporte, ver una película, leer un libro, ir de compras, acudir a un espectáculo o disfrutar del contacto con el medio natural, entre otras múltiples opciones, pueden ser referentes de los tiempos de ocio. Esta diversidad de actividades suele agruparse para su estu-

Fecha recepción: 10-04-19. Fecha de aceptación: 22-10-19 Raúl Fraguela-Vale

fravale@udc.es dio bajo criterios que simplifiquen el análisis de la realidad, reconociéndose cinco dimensiones del ocio (Cuenca, 2014): lúdica (experiencias relacionadas con el juego, incluyendo las actividades físicas en sus diversas manifestaciones), festiva (espacios de encuentro y relación con otras personas), creativa (vivencias culturales relacionadas tanto como con el espectadorismo como con los hobbies), ambientalecológica (recreación al aire libre y turismo) y solidaria (enraizada en la ayuda desinteresada a otras personas, con independencia de la actividad realizada en sí misma).

La importancia del ocio para el colectivo juvenil se ha evidenciado en la investigación reciente (Caballo, Varela \& Nájera, 2017; Caldwell \& Witt, 2011; Gómez-Granell \& Julià, 2015; Guadarrama-Álvarez, 2018; Pronovost, 2013; Roberts, 2014) en tanto que una vivencia plena y satisfactoria de los tiempos libres contribuye al desarrollo de la identidad, la autonomía, el sentimiento de logro o el compromiso social, siendo este período de la vida particularmente apropiado para descubrir nuevos intereses y afirmar los valores personales y otros ideales sociales (Roult, Royer, Auger, \& Adjizian, 2016). Entre las múltiples actividades que realiza la juventud en sus tiempos libres cabe subrayar el poder de atracción que mantiene la práctica deportiva al ocupar un lugar preferente en el ocio juvenil (Fraguela, De-Juanas \& Franco, 2018). En este sentido, la generación de hábitos de práctica de actividad física resulta transcendente para generar estilos de vida activos (y por lo tanto saludables), que pueden permanecer en la vida adulta (Telama, et al., 2014). Sus beneficios 
han sido puestos de manifiesto por diversas fuentes, destacando no solo los referidos a la salud (Hikihara, Watanabe, Kawakatsu \& Ishii, 2018; Román, 2016) o al rendimiento académico (Parker, Astell-Burt \& Lonsdale, 2017), sino también al ámbito social (Monteagudo, et al., 2017).

Si bien a través del deporte se transmiten actitudes y valores de solidaridad, cooperación, integración, etc., también se promueven estereotipos relacionados con el género que vinculan a las mujeres a ciertas prácticas y establecen patrones de actividad claramente diferenciados de los varones. La literatura existente advierte -desde hace décadas- de las diferencias en los hábitos de ocio deportivo en función del género (Fraguela-Vale, Varela-Garrote \& Sanz-Arazuri, 2016; Granda, Montilla, Barbero, Mingorance \& Alemany, 2010; Valdemoros, Ponce de León \& Gradaílle, 2016), manifestándose una preocupante disminución de la actividad físico-deportiva entre las jóvenes en la etapa de Educación Secundaria. En concreto, en los estudios sobre el empleo del tiempo de ocio deportivo en la juventud (Lamoneda \& Huertas, 2017; Lazcano \& Caballo, 2016), el género se presenta como un factor determinante, con diferencias entre chicos y chicas, en aspectos referidos al tiempo y la organización de la práctica, las motivaciones, y el acompañamiento por parte de la familia y las amistades durante la actividad de ocio, entre otras. Respecto al tiempo de práctica, está demostrada la relación entre la participación en actividades físico-deportivas de forma regular y los beneficios sobre la salud, tanto a nivel fisiológico, como psíquico y social (Janssen \& LeBlanc, 2010; Ramos, Rivera, Moreno \& Jiménez-Iglesias, 2012). La práctica de actividad físico-deportiva en la etapa adolescente incrementa la responsabilidad personal (Gómez-Mármol, Sánchez-Alcaraz, De la Cruz, Valero \& González-Víllora, 2017) y la prevención de hábitos nocivos en esta etapa (Chauvet, Martín-Escudero, Martínez-de-Haro, \& Cid-Yagüe, 2018; Guo, Reeder, McGee \& Darling, 2011; Tibbits, Caldwell, Smith, Vergnani \& Wegner, 2016). Sin embargo, tanto en la infancia como en la adolescencia se detectan bajos niveles de actividad físico-deportiva y la presencia de hábitos sedentarios (Vílchez, Ruiz-Juan \& García, 2017).

A pesar de que gran parte de los adolescentes españoles no cumplen las recomendaciones mínimas de práctica de actividad física diaria marcadas por las autoridades sanitarias (García-Moya, Moreno, Rivera, Ramos \& Jiménez-Iglesias, 2012), el nivel de práctica es especialmente reducido entre las chicas (Castillo, Tornero \& García, 2018; GallegosSánchez, Ruiz-Juan, Villareal-Angeles \& Zamarripa, 2019; Lamoneda \& Huertas, 2017). Ellas realizan menos actividad físico-deportiva (Farias, Souza \& Santos, 2016; Práxedes, Sevil, Moreno, Del Villar \& García-González, 2016; Rico-Díaz, Arce-Fernández, Padrón-Cabo, Peixoto-Pino \& AbelairasGómez, 2019; Vega, Saldías \& Viciana, 2017; Vidal-Conti, 2016) $\mathrm{y}$, aunque las diferencias entre ambos grupos son notables a lo largo de la juventud, destaca la edad de los 13 años como un hito en el que se incrementan en mayor medida estas diferencias (Moreno, Ramos, Rivera, Jiménez \& García, 2016; Ramos, Jimenez-Iglesias, Rivera \& Moreno, 2016).

Asimismo, la variable género también condiciona la organización de la práctica de ocio deportivo, puesto que las chicas prefieren practicar por su cuenta, de forma no reglada, mientras que los varones se decantan por la actividad físico- deportiva organizada (Castro-Sánchez, Zurita-Ortega, Martínez-Martínez, Chacón-Cuberos \& Espejo-Garcés, 2016; García-Moya, et al., 2012).

En cuanto a las modalidades deportivas que les atraen, los chicos prefieren los deportes de equipo y de contacto, mientras que las chicas se decantan por actividades de carácter expresivo (como la danza) y por los deportes individuales y sin contacto. Las preferencias de ambos colectivos son claramente diferenciadas (Codina, Pestana, Castillo \& Balaguer, 2016; Hill \& Cleven, 2005), una tendencia que se mantiene en contextos distintos al ocio como el escolar (actividades durante los recreos, asignatura de Educación Física, etc.). En este sentido, Sevil et al. (2015) informan de que mientras los chicos demuestran preferencias por la realización de actividades físicas de cooperación-oposición, más concretamente el fútbol, las chicas están más motivadas para las actividades de carácter expresivo, como el acrosport.

Otra de las variables sobre las que el género tiene un influjo diferenciador son los motivos de práctica del ocio deportivo (Cambronero, Blasco, Chiner \& Lucas, 2015). Una de las principales razones para que las jóvenes realicen actividad físico-deportiva es el cuidado de su imagen corporal, con intención de mejorar su apariencia y controlar su peso. En el caso de las chicas, las motivaciones de práctica son de carácter externo y suelen aludir a una desmotivación hacia la práctica. Los chicos, sin embargo, practican por otros motivos, como son el gusto por la competición, el mantenimiento de su condición física y estar con sus amistades (Egli, Bland, Melton \& Czech, 2011; Marín \& Olivares, 2009; MorenoMurcia, Cervelló-Gimeno \& González-Cutre, 2008; Roberts, Reeves \& Ryrie, 2014). Dado que la motivación en este segundo caso atiende a valores intrínsecos, vinculados a la propia satisfacción de realizar actividad física, aumenta la posibilidad de que estas prácticas se mantengan en el futuro.

Sobre la relevancia del acompañamiento a la práctica, se ha detectado una estrecha asociación entre el ocio deportivo en la adolescencia y la juventud y la existencia en su entorno cercano de un estilo de vida activo, apoyo y refuerzo, tanto por parte de la familia como de los amigos (Hohepa, Scragg, Schofield, Kolt \& Schaaf, 2007). También en este caso se detectan diferencias en función del género, pues mientras las adolescentes prefieren practicar actividad física de forma individual con la intención de mejorar su imagen corporal, los jóvenes de su misma edad realizan actividad físico-deportiva en equipo (por tanto acompañados), salvo aquellos chicos preocupados por su peso corporal que también se decantan por una práctica individual (GonzálezMontero de Espinosa, André, García-Petuya, López-Ejeda, Mora \& Marrodán, 2010).

En este marco, la presente investigación tiene como objetivos analizar las diferencias de género en la práctica de ocio deportivo de la juventud española (15-20 años) y comparar las características de este tipo de práctica entre los chicos y chicas que la identifican como su actividad de ocio preferida. A partir de los objetivos mencionados se desprenden las dos hipótesis que guían este estudio: la primera es que el género continúa siendo una variable decisiva para la comprensión de la práctica de actividades físico-deportivas en la juventud española; la segunda se refiere a que las pre- 
ferencias de género en cuanto al ocio deportivo son muy distintas, especialmente en lo referente al interés por las modalidades que les atraen y la participación en competiciones.

\section{Material y método}

Esta investigación de corte cuantitativo se enmarca dentro de un proyecto coordinado en red realizado por siete universidades españolas dentro del Plan nacional de I+D+i. Para este trabajo se han comparado las características del ocio deportivo de mujeres y hombres jóvenes de nuestro país (15-20 años).

\section{Participantes}

A través de un muestreo probabilístico estratificado mediante afijación proporcional y tomando como referencia territorial las áreas Nielsen y, dentro de éstas, el sexo, el curso y la titularidad de centro, se configuró una muestra representativa de 2694 estudiantes de Educación Secundaria Postobligatoria (error: 1.9\%; nivel de confianza: 95\%). Para el análisis de los patrones de ocio deportivo se seleccionaron aquellos jóvenes que señalaron el deporte como su actividad preferida de ocio (915 personas, 34\% de la muestra).

Las principales características socio-descriptivas de la muestra se presentan en la tabla 1.

\begin{tabular}{|c|c|c|c|c|}
\hline Edad (DT) & Sexo (\%) & $\begin{array}{l}\text { Modalidad de ocio } \\
\text { preferida (\%) }\end{array}$ & Titularidad Centro (\%) & Zonas (\%) \\
\hline $17.15(1.57)$ & $\begin{array}{l}\text { Chico (51.3) } \\
\text { Chica (48.7) }\end{array}$ & $\begin{array}{c}\text { Electrónico (20.1) } \\
\text { Deportivo (30.0) } \\
\text { Cultural (17.5) } \\
\text { Festivo (13.6) } \\
\text { Otro (14.8) }\end{array}$ & $\begin{array}{c}\text { Pública (78.6) } \\
\text { Privada laica (4.5) } \\
\text { Privada religiosa (16.9) }\end{array}$ & $\begin{array}{c}\text { Noreste (17.6) } \\
\text { Levante (12.8) } \\
\text { Sur (28.4) } \\
\text { Centro (22) } \\
\text { Noroeste (11.2) } \\
\quad \text { Norte (8) }\end{array}$ \\
\hline
\end{tabular}

\section{Instrumento}

La obtención de información se realizó a través de la aplicación de un cuestionario elaborado ad hoc para el estudio de los tiempos escolares y de ocio del alumnado de Educación Secundaria Postobligatoria (Bachillerato, FP de Grado medio y PCPI -actualmente FP Básica-). Los bloques temáticos de los que consta son: descripción del alumnado, vida en el centro escolar, vida familiar, tiempo libre, salud y calidad de vida, estudios y mercado laboral en el futuro y emprendimiento.

En este trabajo se presentan los resultados que se corresponden con las siguientes variables, incluidas en los bloques de descripción del alumnado y tiempo libre del cuestionario:

- Modalidades de ocio: las personas participantes seleccionaron de entre 49 actividades de ocio aquella más importante para ellos. Las actividades se agruparon en una variable de 5 categorías: ocio electrónico, deportivo, cultural, festivo y otros ocios.

- Actividades de ocio deportivo: dentro de esta modalidad de ocio, el alumnado podía seleccionar 6 opciones: deporte y actividad física (en general), actividades físicas artísticas y expresivas, en el medio natural, de cooperación y oposición, de oposición uno contra uno e individuales.

- Frecuencia semanal de práctica: aquellos que realiza- ban actividades de ocio deportivo con una frecuencia semanal, tenían que señalar si practicaban 1-2 días, 3-4 días o los 5 días de lunes a viernes. También si lo hacían uno o los dos días del fin de semana.

- Organización de la práctica: se indicó si realizaron las actividades de forma libre (por su cuenta), de forma organizada (en una asociación, club, etc.) o combinando ambas formas.

- Acompañamiento a la práctica: el alumnado especifica si realiza su ocio deportivo en solitario, acompañado por su familia, por gente de su edad o por otras personas.

- Competición: indican si participan o no en competiciones durante su ocio.

- Renuncia a actividades de ocio: informan sobre la existencia o no de actividades de ocio que les gustan a las que tienen que renunciar.

- Motivos de renuncia a actividades de ocio: el alumnado selecciona los motivos por los que renuncia a sus actividades de ocio entre 11 posibles respuestas. Para simplificar el análisis, se agruparon todas aquellas categorías con menos de un 5\% de respuesta en la categoría «otros motivos». Las categorías resultantes fueron las siguientes: falta de tiempo, falta de dinero, demasiadas tareas escolares y otros motivos.

\section{Procedimiento y análisis de datos}

La recogida de datos se realizó en el curso académico 2015-2016. Dos miembros del equipo investigador se desplazaron a cada centro educativo para aplicar los cuestionarios en el aula. El alumnado participó en la investigación de forma anónima y voluntaria, con el conocimiento y consentimiento de las familias y de los equipos directivos, siendo informados de los objetivos de la investigación.

Para estudiar la influencia del género en la selección de las actividades de ocio practicadas (especialmente deportivo) se utilizó la prueba Chi-cuadrado, siendo realizado el tratamiento estadístico mediante el software informático SPSS 22.0. Para el cálculo del tamaño del efecto se calculó el índice w para coeficiente de asociación en tablas de contingencia (en este caso para Chi-cuadrado), a través del programa G*Power 3.1 (Faul, Erdfelder, Lang, \& Buchner, 2007) con potencia (1-â), á = .05.

\section{Resultados}

A continuación se presentan los principales hallazgos obtenidos en la investigación, agrupados en dos bloques temáticos siguiendo los objetivos del estudio: a) diferencias de género en la práctica de ocio deportivo y b) características del ocio deportivo de hombres y mujeres jóvenes.

Diferencias de género en la práctica de ocio deportivo

Con respecto a las modalidades de ocio preferidas, tal y como se indica en la figura 1 , los perfiles de ocio de mujeres y hombres difieren entre sí. Mientras las primeras se decantan en mayor medida que los segundos por los ocios de tipo cultural, festivo y por la categoría de otros ocios, los hombres prefieren el ocio electrónico y deportivo. En ambos colectivos el ocio deportivo es la modalidad más popular. 


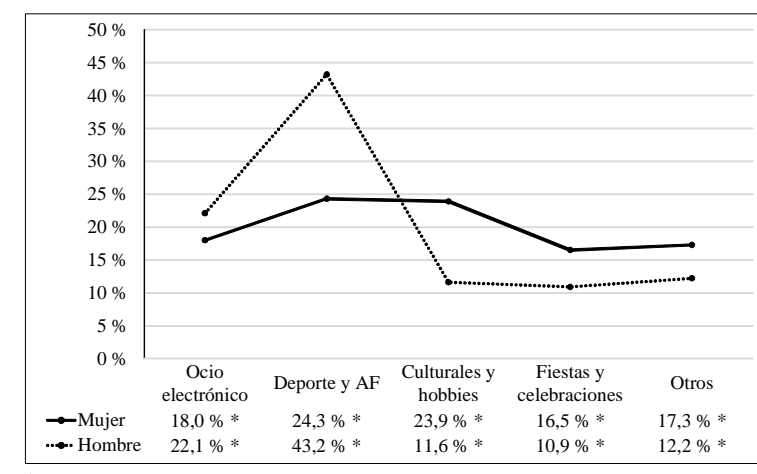

Figura 1 .

Modalidades de ocio preferidas por la juventud española en función del sexo (Chi-Cuadrado). * Valor absoluto residuos tipificados corregidos $>1.9$ (diferencia significativa entre hombres mujeres en esa categoría). Chi-cuadrado=160.907, $p<.001, \mathrm{w}=.28$.

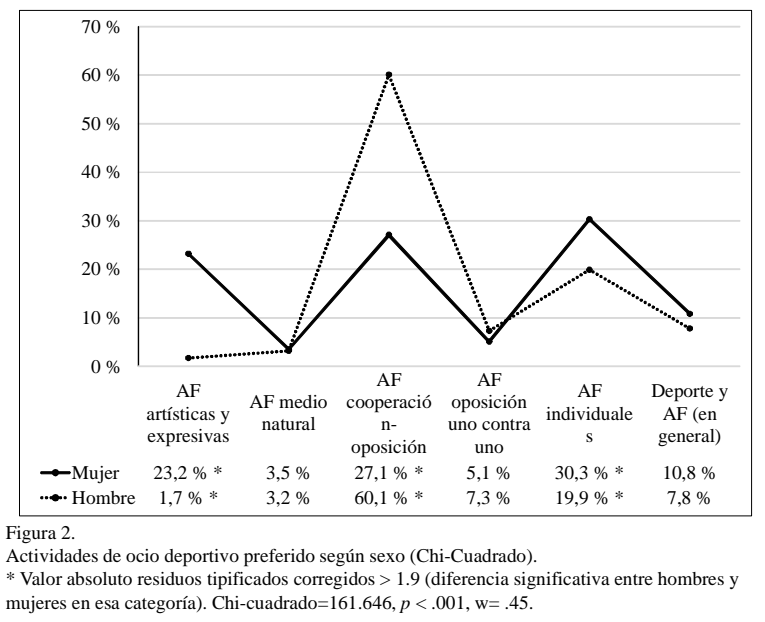

Centrándonos en el ocio deportivo, de las seis categorías de actividades, se encuentran diferencias de género en tres de ellas (figura 2). Las chicas muestran mayor preferencia que los chicos por las actividades artísticas y expresivas y por las individuales, mientras que ellos claramente se decantan por los deportes colectivos competitivos (actividades físicas de cooperación y oposición).

Para completar esta información se estudió la participación en competiciones deportivas de chicos y chicas, destacando la existencia de diferencias significativas (chi-cuadra$\mathrm{do}=5.703, p<.01, \mathrm{w}=.06$ ), dado que ellas participan en menor medida que ellos en este tipo de competiciones (56.6\% vs 64.7\%). Esta tendencia no es exclusiva del ocio deportivo, ya que si se atiende a todos los tipos de ocio, las diferencias de género son todavía más acusadas (chi-cuadrado $=130.726, p$ $<.001$, w= .22), también a favor de los chicos. El tamaño del efecto en ambos casos es pequeño (Cohen, 1988), por lo que la relación entre la implicación en actividades competitivas de ocio y el género debe ser estudiada cuidadosamente y el significado de los resultados interpretados con precaución.

Características del ocio deportivo de mujeres y hombres jóvenes

Con respecto a la frecuencia de práctica, existen diferencias significativas en función del género en la práctica durante la semana (tabla 2). El estudio de los residuos tipificados indica que las chicas realizan actividades de ocio deportivo uno o dos días por semana en mayor medida que los chicos. Lo contrario ocurre en la categoría «3 o 4 días por semana», donde los chicos puntúan significativamente más alto que las chicas. Para la práctica de los 5 días de lunes a viernes, no se detectan diferencias entre ambos colectivos, al igual que respecto a la frecuencia de práctica durante el fin de semana que no presenta diferencias en función del género (tabla 2).

No existen diferencias significativas entre mujeres y hombres en la organización de su ocio deportivo. En concreto, un $51.8 \%$ de la juventud practica de forma organizada, en una asociación, club, etc., un $29.6 \%$ prefiere organizar su ocio deportivo de forma libre, por su cuenta y el $18.6 \%$ restante compagina ambas formas de práctica deportiva. Esta tendencia contrasta con lo que ocurre si analizamos conjuntamente el resto de modalidades de ocio, ya que se identifica una mayor preferencia de las mujeres por las actividades realizadas de forma libre (por su cuenta) y de los hombres por actividades organizadas. En todos los casos el tamaño del efecto es muy pequeño, por lo que el significado de las diferencias debe ser interpretado con precaución.

\begin{tabular}{|c|c|c|c|c|}
\hline & Sexo & $\mathrm{N}$ & $X^{2}$ & $\mathrm{w}$ \\
\hline \multirow{2}{*}{ Ocio deportivo de lunes a viernes } & M & 272 & \multirow{2}{*}{$6.529 *$} & \multirow{2}{*}{.06} \\
\hline & $\mathrm{H}$ & 480 & & \\
\hline Ocio deportivo fines de semana & $\begin{array}{l}\mathrm{M} \\
\mathrm{H}\end{array}$ & $\begin{array}{l}145 \\
308\end{array}$ & .010 & .00 \\
\hline Organización ocio deportivo & M & 289 & 3.779 & .05 \\
\hline \multirow{2}{*}{ Organización ocio (en general) } & M & 897 & \multirow{2}{*}{$6.442^{*}$} & \multirow{2}{*}{.08} \\
\hline & $\mathrm{H}$ & 704 & & \\
\hline
\end{tabular}

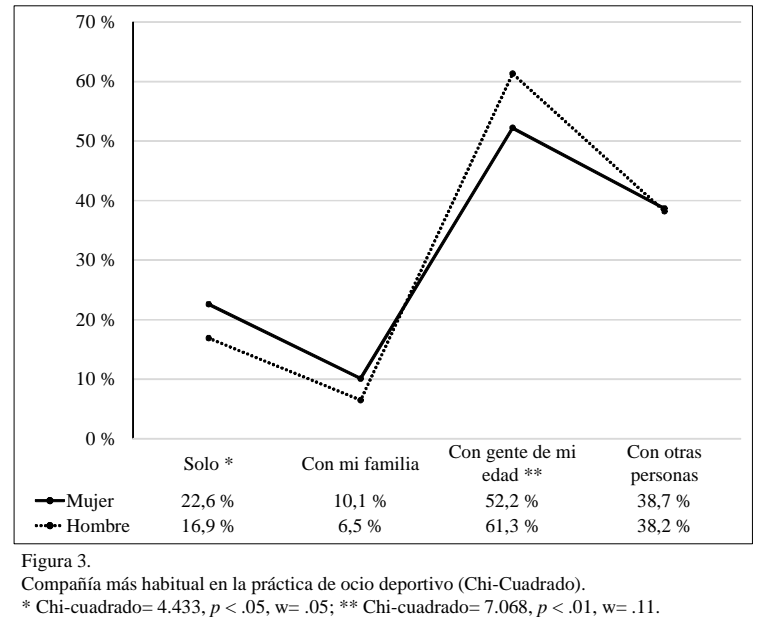

En lo referente a la compañía más habitual en la práctica del ocio deportivo, se detectan diferencias entre hombres y mujeres en dos de los cuatro ítems estudiados (figura 3). Mientras que las chicas tienden a un ocio más solitario que los chicos, estos destacan por compartir la práctica deportiva con sus iguales en mayor medida que las chicas.

Por último, la renuncia a actividades de ocio deportivo que son de su agrado, es habitual para muchas mujeres 44.4\%- y no tanto para los hombres -29.2\%- (chi-cuadra$\mathrm{do}=20.550, p<.001, \mathrm{w}=.16$ ). Esta tendencia no es exclusiva del ocio deportivo, ya que si analizamos conjuntamente las demás modalidades de ocio, las diferencias de género son todavía más acusadas (chi-cuadrado=29.960, $p<.001$, w= .14), siempre con un mayor grado de renuncia por parte de las chicas.

En líneas generales, son múltiples las barreras y motivos de renuncia de las personas jóvenes a actividades de ocio deportivo que les gustaría realizar, destacando entre las principales: la falta de tiempo (40.1\%), la falta de dinero (7.7\%), la 
existencia de demasiadas tareas escolares (10.4\%) y otras causas (41.8\%). Cabe señalar que no se encontraron diferencias significativas en esta cuestión (chi-cuadrado $=6.005, p>$ $.05, \mathrm{w}=.17$ ) dado que aunque renuncian más las chicas que los chicos, ambos colectivos lo hacen por los mismos motivos. También en este caso el tamaño del efecto es pequeño, por lo que el significado de las diferencias debe ser interpretado con cautela.

\section{Discusión}

Los resultados de este estudio ponen de manifiesto la existencia de distintos perfiles de ocio deportivo en función del género. La importancia de la variable género como elemento clave para entender los perfiles de ocio no es nueva (Henderson, 2009; Monteagudo, et al., 2017), si bien es necesario profundizar en las razones que influyen en la toma de decisiones al respecto atendiendo a los intereses y motivaciones de cada colectivo y a las barreras y dificultades en el acceso al ocio, especialmente del sector femenino. En el primer caso, se trata de una característica del ocio fruto de la diversidad y de la expresión de las diferencias de cada género, en las que influyen ineludiblemente patrones de tipo sociocultural que hacen que chicos y chicas se decanten por actividades diferenciadas en la ocupación de sus tiempos libres desde la primera infancia. Los estereotipos de género mantienen un claro reflejo en la práctica físico-deportiva tanto en educación primaria como en secundaria, persistiendo la atribución específica de ciertas actividades a los varones -fuerza, competición- o a las mujeres -belleza, expresión corporal, etc.- (Alvariñas \& Novoa, 2016). A ello se une el miedo al estigma, dado que el hecho de participar en actividades deportivas consideradas poco apropiadas en función el género conlleva el riesgo de ser etiquetado y sentirse amenazado no solo individualmente sino también como miembro de un grupo que no sigue los cánones establecidos (Laurin, 2017).

En segundo lugar, se identifica que las chicas ven limitado su acceso a las actividades preferidas o deseadas debido a condicionantes que no afectan (o lo hacen en menor medida) a sus compañeros del sexo masculino, evidenciándose la incidencia de barreras sociales y educativas en el ejercicio de su derecho al ocio. Los hallazgos son coincidentes con el estudio de Martínez de Quel, Fernández García y Camacho (2010), en el que se apunta que la falta de tiempo es la mayor dificultad percibida a la hora de practicar actividades físicodeportivas, especialmente en la adolescencia femenina; una carencia de tiempo que se atribuye a la intensificación de las labores académicas en la etapa de secundaria y a la existencia de una escala de valores en la que lo realmente importante son los estudios, intensificada por las expectativas de los padres. Bajo este enfoque de análisis de la brecha de género, los datos obtenidos refuerzan la tendencia señalada por la investigación sobre la existencia de una menor participación por parte de las chicas en la competición (Codina, et al., 2016; Hill \& Cleven, 2005; Sevil, et al., 2015) y por un mayor interés en modalidades deportivas, como las actividades artísticas y expresivas y las individuales.

Respecto a la frecuencia de práctica, se encuentra una menor intensidad de participación en las chicas que en los chicos durante la semana (de lunes a viernes), que es precisamente cuando se dispone de menos tiempo. Puede interpretarse que en situaciones de escasa disponibilidad temporal, las mujeres renuncian en mayor medida a la práctica deportiva que los hombres. Sin embargo, cuando disponen de más tiempo (durante los fines de semana) ambos grupos presentan una frecuencia de práctica deportiva similar (Beltrán, Sierra, Jimenez, González-Cutre, Martinez \& Cervelló, 2017; Macarro, Romero \& Torres, 2010). Esto parece indicar que la menor frecuencia se debe más a factores externos (principalmente la falta de tiempo) que a un menor interés, gusto o motivación por la práctica físico-deportiva entre las chicas. Además, esta tendencia se ve reforzada por los datos aportados sobre la renuncia a las actividades deportivas de ocio, significativamente más altos para las mujeres que para los hombres (Caballo, et al., 2017), con la falta de tiempo como un elemento destacado entre los motivos de dicha renuncia (Sevil, Praxedes, Zaragoza, Del Villar \& GarcíaGonzález, 2017).

La forma predominante de organización del ocio deportivo es de carácter asociativo, destacando que tanto las chicas como los chicos prefieren practicar deporte en asociaciones y clubes que hacerlo por su cuenta. Este hallazgo resalta el carácter social y organizado que caracteriza al deporte frente a otras prácticas menos estructuradas y que, según los datos de este estudio, predominan en los tiempos libres de las mujeres, como las culturales y las festivas. Cabe señalar que el carácter organizado de las actividades de ocio tiene un efecto positivo sobre el compromiso e integración social de las personas al favorecer la continuidad y la frecuencia de práctica, frente a otras más discontinuas y ocasionales (Isorna, Rial \& Vaquero-Cristóbal, 2014; Romero, Garrido \& Zagalaz, 2009).

Del análisis del acompañamiento en la práctica del ocio deportivo se deduce que ambos colectivos se decantan mayoritariamente por compartir las actividades con gente de su edad. Sin embargo, las chicas practican en soledad en mayor medida que los chicos y éstos superan significativamente a las chicas en la práctica con sus iguales. Como se ha indicado con anterioridad, existen dos líneas argumentales complementarias para explicar esta realidad: de un lado, la propia tipología de las actividades preferidas por chicas y chicos y, de otro, las distintas motivaciones de práctica deportiva en estas edades (González-Montero de Espinosa, et al., 2010).

En consecuencia, se concluye que las características y preferencias en la vivencia del ocio en general y del ocio deportivo en particular difieren notablemente entre mujeres y hombres, haciéndose necesarias nuevas investigaciones que posibiliten definir cuáles de estas diferencias deben atribuirse a decisiones libres y conscientes de las personas y cuáles derivan de desventajas o barreras de ocio que condicionan el acceso a las prácticas deseadas, especialmente entre las jóvenes, incorporando una dimensión cualitativa. Desde esta perspectiva, el estudio que se presenta intenta aportar información que ayude a comprender por qué a pesar del unánime acuerdo internacional sobre los beneficios de la práctica de actividad físico-deportiva en los tiempos libres de la juventud, la brecha de género se mantiene, década tras década y por abrumadora diferencia, como la más 
amplia entre todas las modalidades de ocio estudiadas, siendo la adolescencia un periodo crítico.

Ante estos hallazgos, se advierte de la responsabilidad de los centros educativos en la promoción de hábitos de vida activos y saludables entre su alumnado en clave de género desde una doble perspectiva: de un lado, sus dinámicas cotidianas deben generar oportunidades de práctica dentro del horario lectivo y fuera de él (actividades físico-deportivas extraescolares) y, de otro, las distintas materias, y especialmente la Educación Física, deben contribuir a aumentar la autopercepción de competencia motriz de todo el alumnado, factor positivamente ligado a los niveles de práctica de actividad físico-deportiva, tanto en el contexto lectivo como en el de ocio (Garcia, Rodríguez, Pérez, Rosa \& López, 2015). Además, con el horizonte de superar las desigualdades de género en el contexto escolar, se hace necesario la puesta en marcha de metodologías cooperativas e inclusivas que favorezcan la participación equitativa de chicos y chicas en actividades deportivas (Lamoneda \& Huertas, 2017), de modo que el profesorado trate de romper con los estereotipos cuidando la utilización del lenguaje, buscando agrupaciones mixtas que varíen periódicamente, asegurándose que los alumnos no ocupan lugares más favorables, más grandes y/o mejor ubicados que las alumnas (Rodríguez \& Miraflores, 2018).

No obstante, aun desempeñando la escuela un papel esencial, la responsabilidad educativa no se limita a ella, sino que la superación de los estereotipos relacionados con el género exige del compromiso ineludible de diversos agentes como las familias, los servicios de ocio, las administraciones locales o los medios de comunicación. La conformación de los gustos e intereses sobre los que se asientan las experiencias de ocio deportivo y las oportunidades que la juventud tiene para poder vivenciarlas transciende ampliamente los contextos escolares, delimitándose en los múltiples escenarios en los que transcurre su vida cotidiana, siendo la brecha de género un factor esencial a considerar.

Como toda investigación, este trabajo presenta limitaciones, vinculadas principalmente a la ausencia de medidas objetivas de actividad física que completen las preferencias expresadas por los participantes sobre su ocio deportivo. Esto hace que los perfiles de ocio se analicen exclusivamente en base a la percepción de los sujetos estudiados. Se propone como futura línea de investigación contrastar la información aportada por los participantes con mediciones objetivas de su actividad física en un determinado período de tiempo, a fin de identificar la evolución de sus prácticas deportivas según el género.

\section{Agradecimientos}

Los autores desean agradecer a los jóvenes su participación en este estudio y a los centros escolares que facilitaron la realización del mismo. El presente trabajo es resultado de los siguientes proyectos de investigación:

- «De los tiempos educativos a los tiempos sociales: la construcción cotidiana de la condición juvenil en una sociedad de redes. Problemáticas específicas y alternativas pedagógico-sociales» (Proyecto Coordinado EDU 2012-39080C07-00) y del subproyecto: «De los tiempos educativos a los tiempos sociales: la construcción cotidiana de la condición juvenil en una sociedad de redes: Problemáticas específicas y alternativas pedagógico-sociales» (EDU 2012-39080-C0701), cofinanciados en el marco del plan nacional I+D+i con cargo a una ayuda del Ministerio de Economía y Competitividad, y por el Fondo Europeo de Desarrollo Regional(FEDER, 2007-2013).

- «Educar el ocio: realidades y perspectivas en clave intergeneracional, integral e inclusiva en una sociedad de redes» (Proyecto EDU2015-65638-C6-1-R), financiado en el marco del plan nacional $\mathrm{I}+\mathrm{D}+\mathrm{i}$ con cargo a una ayuda del Ministerio de Economía y Competitividad.

\section{Referencias}

Alvariñas, M. \& Novoa, A. (2016). Pensamientos relacionados con la actividad física y el género en adolescentes de Galicia. Sportis, 2(1), 23-35. doi:10.17979/ sportis.2016.2.1

Beltrán, V.J., Sierra, A.C., Jimenez, A., González-Cutre, D., Martinez, C. \& Cervelló, E. (2017). Diferencias según género en el tiempo empleado por adolescentes en actividad sedentaria y actividad física en diferentes segmentos horarios del día. RETOS. Nuevas Tendencias en Educación Física, Deporte y Recreación, 31, 3-7.

Caballo, M.B., Varela, L. \& Nájera, E.M. (2017). El ocio de los jóvenes en España. Una aproximación a sus prácticas y barreras. OBETS. Revista de Ciencias Sociales, 12(Extra 1), 43-64. doi:10.14198/OBETS2017.12.1.11.

Caldwell, L. \& Witt, P. (2011). Leisure, recreation and play from a developmental context. New Directions for Youth Development, 130, 13-27.

Cambronero, M., Blasco, J.E., Chiner, E. \& Lucas, A.G. (2015). Motivos de participación de los estudiantes universitarios en actividades físico-deportivas. Revista Iberoamericana de Psicología del Ejercicio y el Deporte, 10(2), 179-186.

Castillo, E., Tornero, I. \& García, J.A. (2018). Relación entre actividad física, alimentación y familia en edad escolar. RETOS. Nuevas Tendencias en Educación Física, Deporte y Recreación, 34, 85-88.

Castro-Sánchez, M., Zurita-Ortega, F., Martínez-Martínez, A., Chacón-Cuberos, R. \& Espejo-Garcés, T. (2016). Clima motivacional de los adolescentes y su relación con el género, la práctica de actividad física, la modalidad deportiva, la práctica deportiva federada y la actividad física familiar. RICYDE: Revista Internacional de Ciencias del Deporte, 12(45), 262-277. doi: 10.5232/ ricyde2016.04504.

Chauvet, M., Martín-Escudero, M.P., Martínez-de-Haro, V. \& Cid-Yagüe, L. (2018). Actividad física y hábito tabáquico en estudiantes adolescentes. Revista Internacional de Medicina y Ciencias de la Actividad Física y el Deporte, 18(69), 151-164. doi: 10.15366/ rimcafd2018.69.010.

Codina, N., Pestana, J.V., Castillo, I. \& Balaguer, I. (2016). Ellas a estudiar y bailar, ellos a hacer deporte: un estudio de las actividades extraescolares de los adolescentes mediante los presupuestos de tiempo. Cuadernos de Psicología del Deporte, 16(1), 233-242. 
Cohen, J. (1988). Statistical power analysis for the behavioral sciences (2nd ed.). New York: Routledge.

Cuenca, M. (2014). Ocio valioso. Bilbao: Universidad de Deusto.

Dumazedier, J. (1964). Hacia una civilización del ocio. Barcelona: Estela.

Egli, T., Bland, H.W., Melton, B.F. \& Czech, D.R. (2011). Influence of Age, Sex, and Race on College Students' Exercise Motivation of Physical Activity. Journal of american college health, 59(5), 399-406. doi: 10.1080/ 07448481.2010.513074.

Farias, E.S., Souza, L.G. \& Santos, J.P. (2016). Estilo de vida de escolares adolescentes. Adolesc Saude, 13(2), 40-49.

Faul, F., Erdfelder, E., Lang, A., \& Buchner,A. (2007). G*power 3: A flexible Statistical power analysis program for the social, behavioral, and biomedical sciences. Behavior Research Methods, 39(2), 175-191. doi: 10.3758/ bf03193146.

Fraguela-Vale, R., Varela-Garrote, L. \& Sanz-Arazuri, E. (2016). Ocio deportivo, imagen corporal y satisfacción vital en jóvenes españoles. Revista de Psicología del Deporte, 25(2), 33-38.

Fraguela, R., De-Juanas, A. \& Franco, R. (2018). Ocio deportivo en jóvenes potencialmente vulnerables: beneficios percibidos y organización de la práctica. Pedagogía Social. Revista Interuniversitaria, 31, 49-58. doi: 10.7179/ PSRI_2018.31.04

Gallegos-Sánchez, J.J., Ruíz-Juan, F., Villarreal-Angeles, M.A. \& Zamarripa, J.I. (2019). Etapas de cambio en la práctica de actividad física de tiempo libre en estudiantes de secundaria de Victoria de Durango, México. RETOS. Nuevas Tendencias en Educación Física, Deporte y Recreación, 35, 196-200.

García-Moya, I., Moreno, C., Rivera, F., Ramos, P. \& JiménezIglesias, A. (2012). Iguales, familia y participación en actividades deportivas organizadas durante la adolescencia. Revista de Psicología del Deporte, 21(1), 153-158.

Garcia, E., Rodríguez, P., Pérez, J., Rosa,A. \& López, F. (2015). Autopercepción de competencia motriz y su relación con la práctica físico-deportiva en escolares de la Región de Murcia (España). REXE. Revista de Estudios y Experiencias en Educación, 14(27), 49-61.

Gómez-Granell, C. \& Julià, A. (2015). Tiempo de crecer, tiempo para crecer. Barcelona: Ajuntament de Barcelona.

Gómez-Mármol, A., Sánchez-Alcaraz, B.J., De la Cruz, E., Valero, A. \& González-Víllora, S. (2017). Personal and social responsibility development through sport participation in youth scholars. Journal of Physical Education and Sport, 17(2), 775-782. doi: 10.7752/ jpes.2017.02118.

González-Montero de Espinosa, M., André, A.L., GarcíaPetuya, E., López-Ejeda, N., Mora, A.I. \& Marrodán, M.D. (2010). Asociación entre actividad física y percepción de la imagen corporal en adolescentes madrileños. Nutrición Clínica y Dietética Hospitalaria, 30(3), 4-12.

Granda, J., Montilla, M., Barbero, J.C., Mingorance, A. \& Alemany, I. (2010). Frecuencia de práctica y motivos de participación/no participación en actividades físicas en función del género de escolares de 10-12 años de Melilla. RICYDE: Revista Internacional de Ciencias del Deporte, 6(21), 280-296. doi:10.5332/ricyde2010.02103.
Guadarrama-Álvarez, L. (2018). Un acercamiento a las preferencias ocio-recreativas de los jóvenes en el contexto santiaguero. Santiago, número especial, 214-226.

Guo, H., Reeder, A., McGee, R. \& Darling, H. (2011). Adolescents' leisure activities, parental monitoring and cigarette smoking - a cross-sectional study. Substance Abuse Treatment, Prevention, and Policy, 6(12),1-4. doi: 10.1186/1747-597X-6-12.

Henderson, K.A. (2009). Just research and physical activity: Diversity is more than an independent variable. Leisure Sciences, 31(1), 100-105.

Hikihara, Y., Watanabe, M., Kawakatsu, S. \& Ishii, K. (2018). Benefits of organized sports participation and voluntary outdoor play in children and adolescents based on evidence-related health outcomes. Japanese Journal of Physicial Fitness and Sports Medicine, 67(1), 83-98.

Hill, G. \& Cleven, B. (2005). A comparison of 9th grade male and female physical education activities preference and support for coeducational groupings. The Physical Educator, 62(4), 187-198.

Hohepa, M., Scragg, R., Schofield, G., Kolt, G. \& Schaaf, D. (2007). Social support for youth physical activity: Importance of siblings, parents, friends and school support across a segmented school day. Internatio-nal Journal of Behavioral Nutrition and Physical Activity, 4(54).

Isorna, M., Rial, A. \& Vaquero-Cristóbal, R. (2014). Motivaciones para la práctica deportiva en escolares federados y no federados. RETOS. Nuevas Tendencias en Educación Física, Deporte y Recreación, 25, 80-84.

Janssen, I. \& LeBlanc, A. (2010). Systematic review of the health benefits of physical activity and fitness in schoolaged children and youth. International Journal of Behavioral Nutrition and Physical Activity, 7(14). doi: 10.1186/1479-5868-7-40.

Lamoneda, J \& Huertas, F.J. (2017). Análisis de la práctica deportiva-recreativa, tipo de interacción motriz, uso del espacio e instalación en adolescentes en función del género. RETOS. Nuevas Tendencias en Educación Física, Deporte y Recreación, 32, 25-29.

Laurin, R. (2017). Group and individual stereotype threat and identity management strategies: An investigation of rugby women. Journal of Applied Social Psychology, 47(10), $1-5$.

Lazcano, I. \& Caballo, M.B. (2016). Ocio deportivo juvenil: relación entre satisfacción e implicación organizativa. Revista de Psicología del Deporte, 2(2), 9-14.

Macarro, J., Romero, C. \& Torres, J. (2010). Motivos de abandono de la práctica de actividad físico-deportiva en los estudiantes de Bachillerato de la provincia de Granada. Revista de Educación, 353, 495-519.

Marín, F.J. \& Olivares, A. (2009). Motivaciones hacia la práctica física deportiva y su entorno social en el alumnado de secundaria de un centro público y un centro privado de Granada. RETOS. Nuevas Tendencias en Educación Física, Deporte y Recreación, 16, 108-110.

Martínez de Quel, O., Fernández García, E. \& Camacho, M.J. (2010). Percepción de dificultades para la práctica de actividad física en chicas adolescentes y su evolución con la edad. Apunts. Educación Física y Deportes, 99, 9299. 
Monteagudo, M.J., Ahedo, R. \& Ponce de León, A. (2017). Los beneficios del ocio juvenil y su contribución al desarrollo humano. OBETS. Revista de Ciencias Sociales, 12(1), 177-202. doi: 10.14198/OBETS2017.12.1.17.

Monteagudo, M. J. \& Cuenca, M. (2012). Los itinerarios de ocio desde la investigación: tendencias, retos y aportaciones. Pedagogía Social. Revista Interuniversitaria, 19,103-135.doi: 10.7179/PSRI_2012.20.03.

Moreno-Murcia, J.A., Cervelló-Gimeno, E. \& González-Cutre, D. (2008). Relationships among goal orientations, motivational climate and flow in adolescent athletes: differences by gender. The Spanish Journal of Psychology, 11,181-191.doi: 10.1017/S1138741600004224.

Moreno, M.C., Ramos, P., Rivera, F.J., Jiménez, A.M. \& García, I. (2016). Los adolescentes españoles: estilos de vida, salud, ajuste psicológico y relaciones en sus contextos de desarrollo. Resultados del Estudio HBSC-2014 en España: Ministerio de Sanidad.Servicios Sociales e Igualdad.

Ortega, C., Lazcano, I. \& Rocha, M.M. (2015). Espacios de ocio para jóvenes, de la monitorización a la autogestión. Pedagogía Social. Revista Interuniversitaria, 25, 6989. doi:10.7179/PSRI_2015.25.04.

Parker, S., Astell-Burt, T. \& Lonsdale, C. (2017). Regular physical activity and educational outcomes in youth: a longitudinal study. Journal of Adolescent Health, 62(3), 334-340. doi: 10.1016/j.jadohealth.2017.09.014.

Práxedes, A., Sevil, J., Moreno, A., Del Villar, F. \& GarcíaGonzález, L. (2016). Niveles de actividad física en estudiantes universitarios: Diferencias en función del género, la edad y los estados de cambio. Revista Iberoamericana de Psicología del Ejercicio y el Deporte, 11(1), 123-132.

Pronovost, G. (2013). Comprendre les jeunes d'aujourd'hui. Trajectoires, temporalités (Understanding Today's Youth. Trajectories, Timeframes). Sainte-Foy: Presses de l'Université du Québec.

Ramos, P., Jimenez-Iglesias, A., Rivera, F. \& Moreno, C. (2016). Physical activity trends in spanish adolescents. Revista Internacional De Medicina Y Ciencias De La Actividad Fisica Y Del Deporte, 16(62), 335-353. doi: 10.15366/ rimcafd2016.62.010.

Ramos, P., Rivera, R., Moreno, C. \& Jiménez-Iglesias, A. (2012). Análisis de clúster de la actividad física y las conductas sedentarias de los adolescentes españoles, correlación con la salud biopsicosocial. Revista de Psicología del Deporte, 21(1), 99-106.

Rico-Díaz, J., Arce-Fernández, C., Padrón-Cabo, A.., PeixotoPino, L. \& Abelairas-Gómez, C. (2019). Motivaciones y hábitos de actividad física en alumnos universitarios. RETOS. Nuevas Tendencias en Educación Física, Deporte y Recreación, 36, 446-453.

Roberts, K. (2014). Youth and leisure in an age of austerity. In C. Ortega \& F. Bayón (Eds.), El papel del ocio en la construcción social del joven (pp. 17-31). Bilbao: Universidad de Deusto.

Roberts, S., Reeves, M. \& Ryrie, A. (2014). The influence of physical activity, sport and exercise motives among UK based university students. Journal of Further and Higher Education. doi: 10.1080/0309877X.2014.938265.
Rodríguez Rodríguez, L. \& Miraflores, W. (2018). Propuesta de igualdad de género en Educación Física: adaptaciones de las normas en fútbol. RETOS. Nuevas tendencias en Educación Física, Deporte y Recreación, 33, 293297.

Román, B. (2016). Informe 2016: actividad física en niños y adolescentes en España. Barcelona: Fundación para la investigación nutricional.

Romero, S., Garrido, M. \& Zagalaz, M. (2009). El comportamiento de los padres en el deporte. RETOS. Nuevas Tendencias en Educación Física, Deporte y Recreación, 15, 29-34.

Roult, R., Royer, C., Auger, D. \& Adjizian, J.M. (2016). Development of adolescents' leisure interests and social involvement: perspectives and realities from youth and local stakeholders in Quebec. Annals of leisure research, 19(1), 47-61. doi: 10.1080/11745398.2015.1031805.

Sevil, J., Abós, Á., Julián, J.A., Murillo, B. \& García-González, L. (2015). Género y motivación situacional en educación física: Claves para el desarrollo de estrategias de intervención. RICYDE: Revista Internacional de Ciencias del Deporte, 11(41), 281-296. doi: 10.5232/ ricyde2015.04106.

Sevil, J., Praxedes, A., Zaragoza, J., Del Villar, F. \& GarcíaGonzález, L. (2017). Barreras percibidas para la práctica de actividad física en estudiantes universitarios. Diferencias por género y niveles de actividad física. Universitas Psychologica, 16(4), 1-15.

Telama, R., Yang, X., Leskinen, E., Kankaanpa“a”, A., Hirvensalo, M., Tammelin, T. \& Raitakari, O.T. (2014). Tracking of physical activity from early childhood through youth into adulthood. Medicine and Science in Sports and Exercise, 46(5), 955-962. doi: 10.1249/ MSS.0000000000000181.

Tibbits, M.K., Caldwell, L.L., Smith, E.A., Vergnani, T. \& Wegner, L. (2016). Longitudinal Patterns of Active Leisure among South African Youth: Gender Differences and Associations with Health Risk Behaviours. World leisure journal, 58(1), 60-68.

Ureta, X. (2015). Estilos de vida y prácticas de ocio en estudiantes de la Licenciatura en Educación Física, Recreación y Deporte del IUACJ. Revista Universitaria de la Educación Física y el Deporte, 8(8), 49-58.

Valdemoros, M.A., Ponce de León, A. \& Gradaílle, R. (2016). Actividad física de ocio juvenil y desarrollo humano. Revista de Psicología del Deporte, 25(2), 45-51.

Vega, D.M., Saldías, M. P. \& Viciana, J. (2017). Comparison of moderate-to-vigorous physical activity levels between physical education, school recess and after-school time in secondary school students: An accelerometer-based study. Kinesiology, 49(2), 242-251.

Vidal-Conti, J. (2016). Identification of physical activity predictors in schoolchildren according to a socioecological model using multifactorial analysis. Cultura, Ciencia y Deporte, 11(31), 51-59. doi: 10.12800/ ccd.v11i31.642.

Vílchez, M.P., Ruiz-Juan, F. \& García, M.E. (2017) Estudio transcultural de la percepción de competencia escolar y tiempo de ocio. Revista Internacional de Medicina y Ciencias de la Actividad Física y el Deporte, 17(67), 573-587.doi:10.15366/rimcafd2017.67.012 